\title{
Payload Accommodations in NASA's Space Launch System, Block 1 and Beyond
}

\author{
Andrew A. Schorr ${ }^{1}$ and Dr. Kimberly F. Robinson ${ }^{2}$ \\ NASA's Space Launch System Program, Marshall Space Flight Center, AL, 35812 USA \\ Beverly A. Perry ${ }^{3}$ \\ NASA's Space Launch System Program, Marshall Space Flight Center, AL, 35812 USA
}

\begin{abstract}
As part of NASA's new deep space exploration system, the Space Launch System (SLS) will provide the United States with guaranteed access to deep space and an unparalleled capability for launching primary and co-manifested payloads beyond Earth's orbit. Planned missions for the new SLS family of vehicles include launching the Orion spacecraft and elements of the new Gateway astronaut-tended outpost to lunar orbit and sending robotic probes deep into the solar system, such as to Jupiter's moon Europa. If mission parameters allow, secondary payloads in $6 \mathrm{U}, 12 \mathrm{U}$ or larger sizes will also have rideshare opportunities, providing CubeSats with access to deep space. The SLS vehicle will evolve into progressively more powerful variants with fairings in several sizes available to meet an array of mission needs. Superior mass, volume and characteristic energy (C3) enable sending larger, heavier payloads to a variety of destinations. Several elements of the Block 1 vehicle for the first mission, Exploration Mission-1 (EM-1) are complete and have been delivered to the Exploration Ground Systems (EGS) Program at Kennedy Space Center (KSC), which has responsibility for integrating and launching the vehicle. Contractors are already at work manufacturing the second Block 1 vehicle and incorporating numerous lessons learned in manufacturing America's first super heavy-lift deep space rocket since the Apollo Program's Saturn V enabled humankind to take a giant leap forward.
\end{abstract}

\section{Introduction}

With a generation of missions to low-Earth orbit (LEO) under its belt, NASA's Human Exploration and Operations Mission Directorate (HEOMD) is now planning missions to the Moon in order to design, develop, test and evaluate new technologies that will be needed for extended human missions to deep space and eventually Mars. With its ambitious new deep space exploration portfolio - the Space Launch System (SLS), Orion and Exploration Ground Systems (EGS) - and partnerships with commercial companies, NASA will have capability to return astronauts to lunar orbit, assemble and operate a new outpost in cislunar space and send robotic missions to the lunar surface and deep into the solar system - perhaps even to interstellar space.

The cornerstone capability to enable a generation of missions to deep space is SLS, NASA's new super heavy-lift launch vehicle, managed out of NASA's Marshall Space Flight Center (MSFC) in Huntsville, Alabama, USA. With its unmatched performance and flexible architecture, SLS gives NASA a vehicle that will evolve to progressively more powerful configurations and provide unmatched mass and volume to orbit, allowing game-changing missions to deep space. In addition, greater departure energy can reduce transit times when sending robotic probes into the outer solar system or allow for greater mass to orbit, depending on the mission.

\section{A. Sustained Lunar Presence}

NASA inspired the world when the first humans walked on the Moon nearly 50 years ago as part of the Apollo Program. Since then, NASA's human spaceflight program has primarily focused on establishing a permanent human presence in LEO with the International Space Station (ISS). Now, at the dawn of a new decade, NASA plans to return to the Moon - this time to stay. To make a sustained lunar presence possible, NASA plans to build an astronaut-tended

\footnotetext{
${ }^{1}$ Deputy Manager, Spacecraft Payload Integration \& Evolution (SPIE) Office

${ }^{2}$ Payloads Manager, Spacecraft Payload Integration \& Evolution (SPIE) Office

${ }^{3}$ Communications Strategist, Strategic Communications Office
} 
outpost known as the Gateway, notionally consisting of at least one habitat module, a power and propulsion element, an airlock, a robotic arm and a spacewalk capability. Crew, which will be deployed on 30- to 60day missions, will perform science, assess habitability and investigate exploration technologies that require a deep space environment for evaluation. The Agency's overarching plan is for a partnership with commercial launch vehicles to launch smaller payloads and provide service and logistics flights to the Gateway while the superior capabilities for launching mass and volume that SLS provides will enable the Agency to send astronauts in Orion to the Gateway, along with large-volume co-manifested payloads. While conducting scientific study of the Moon with capabilities that can evolve for Mars exploration, NASA's investments in deep space exploration technologies will buy down risk and enable sustained lunar surface operations.

\section{SLS: NASA's Next- Generation Moon Rocket}

For the SLS Program, vehicle designers have planned a family of rockets that will evolve to provide maximum performance for sending strategic payloads to deep space. SLS is planned to be available in crew and cargo configurations, with the first variant, Block 1, capable of sending at least 26 metric tons (t) to trans-lunar injection (TLI) and utilizing a 5 meter $(\mathrm{m})$ fairing in its cargo configuration. The second variant, Block 1B, will use a more powerful Exploration Upper

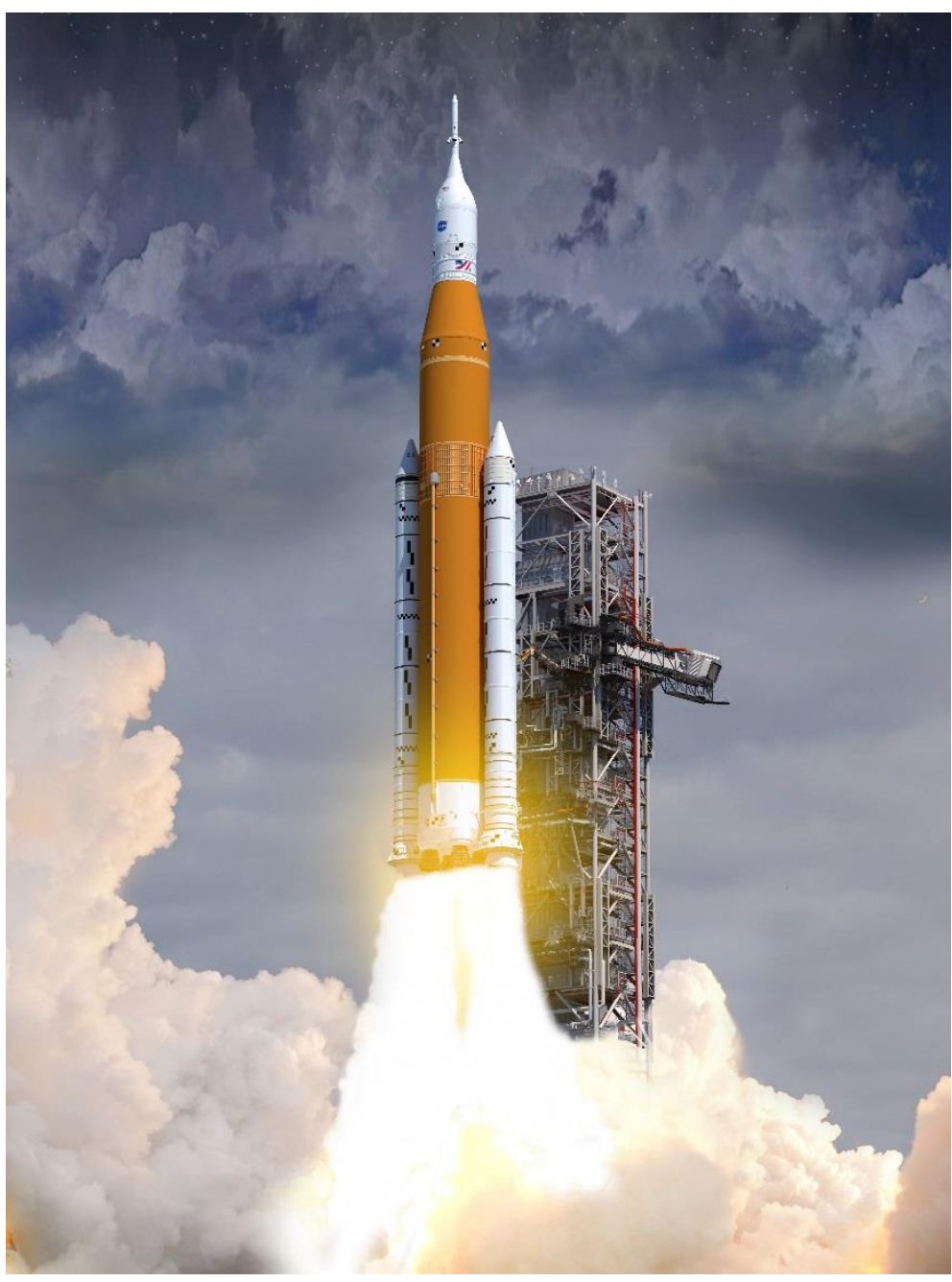

Figure 1. The Space Launch System (SLS) is the cornerstone launch vehicle of NASA's new deep space exploration system, which will return astronauts to lunar orbit for the first time since the Apollo Program. Stage (EUS) to lift at least $37 \mathrm{t}$ to TLI and utilize $8.4 \mathrm{~m}$ fairings in varying lengths. The Block 1B crew vehicle will also have volume for co-manifested payloads in a Universal Stage Adapter (USA). For Mars-class payloads, the Block 2 vehicle is anticipated to have the capability to lift at least $45 \mathrm{t}$ to TLI. Larger fairing sizes are under study for the Block 2 cargo configuration.

The initial variant, Block 1, is planned for the first three missions. Exploration Mission-1 (EM-1) will be the first integrated mission of SLS and Orion and it will launch from modernized facilities at Kennedy Space Center (KSC). EM-1's primary objective will be to verify and validate new systems and technologies of the deep space exploration system, including Orion's new heat shield. After the primary mission is complete, $136 \mathrm{U}$ secondary payloads from a range of payload developers in academia, industry and government, as well as winners of the ground portion of NASA's Centennial Challenges Cube Quest competition, will be released along the upper stage disposal trajectory.

The SLS architecture is comprised of flight-proven propulsion elements: twin solid rocket boosters manufactured by prime contractor Northrop Grumman and four RS-25 liquid hydrogen (LH2)-liquid oxygen (LOX) engines made by Aerojet Rocketdyne. The central core stage, an all-new development, is being manufactured by Boeing at the 


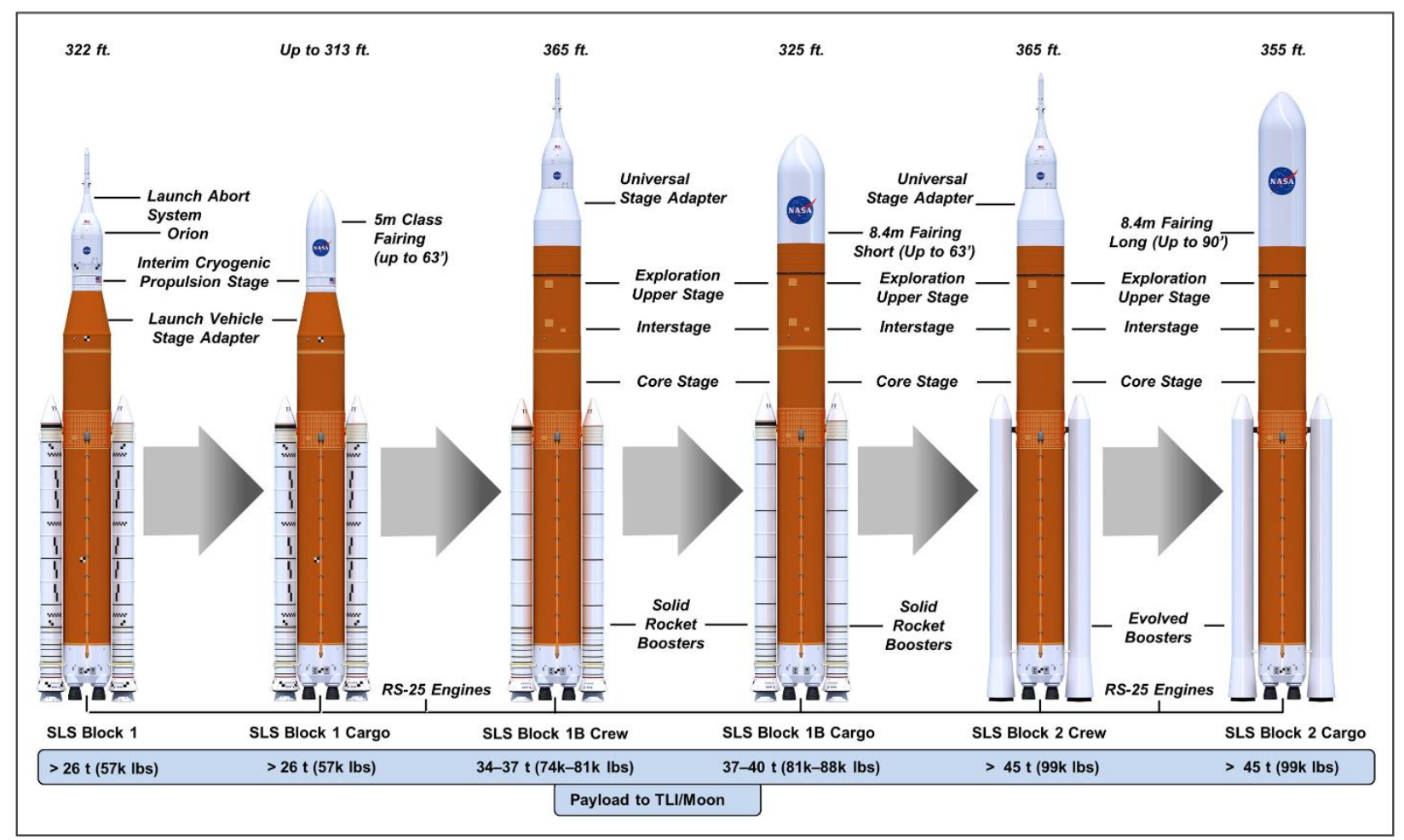

Figure 2. With an evolvable path forward, the SLS family of vehicles will be available in crew and cargo configurations and offer unmatched performance.

historic Michoud Assembly Facility near New Orleans, Louisiana, USA. Above the core stage, an upper stage known as the Interim Cryogenic Propulsion Stage (ICPS), based on the United Launch Alliance (ULA) Delta Cryogenic Second Stage (DCSS), performs the TLI burn during EM-1 that sends Orion toward the Moon. The ICPS is partially covered by a Launch Vehicle Stage Adapter (LVSA) and another adapter, the Orion Stage Adapter (OSA), connects the ICPS to a spacecraft adapter and Orion's Service Module.

Following EM-1, Block 1 will be used for the first crewed mission to lunar orbit, Exploration Mission-2 (EM-2). In addition, the Block 1 cargo variant with a $5 \mathrm{~m}$-class fairing is under consideration to launch Science Mission-1 (SM-1), the "Europa Clipper" mission, on a direct trajectory to the icy Jovian moon.

\section{SLS Payload Accommodations}

The SLS family of vehicles, with superior payload accommodations, can meet a wide array of mission needs. Greater mass, volume and characteristic energy (C3) enable sending larger, heavier payloads to a variety of destinations. Fairings in the $5 \mathrm{~m}$ class will comfortably accommodate the largest science payloads currently under development. For Block 1B and Block 2 cargo configurations, vehicle planners are evaluating $8.4 \mathrm{~m}$ fairings in $62.7 \mathrm{ft} .(19.1 \mathrm{~m})$ and $90 \mathrm{ft} .(27.4 \mathrm{~m})$ lengths. The SLS payload accommodations are largely built on industry-standard interfaces, such as those offered by Evolved Expendable Launch Vehicles (EELVs), enabling payload developers to utilize existing designs and

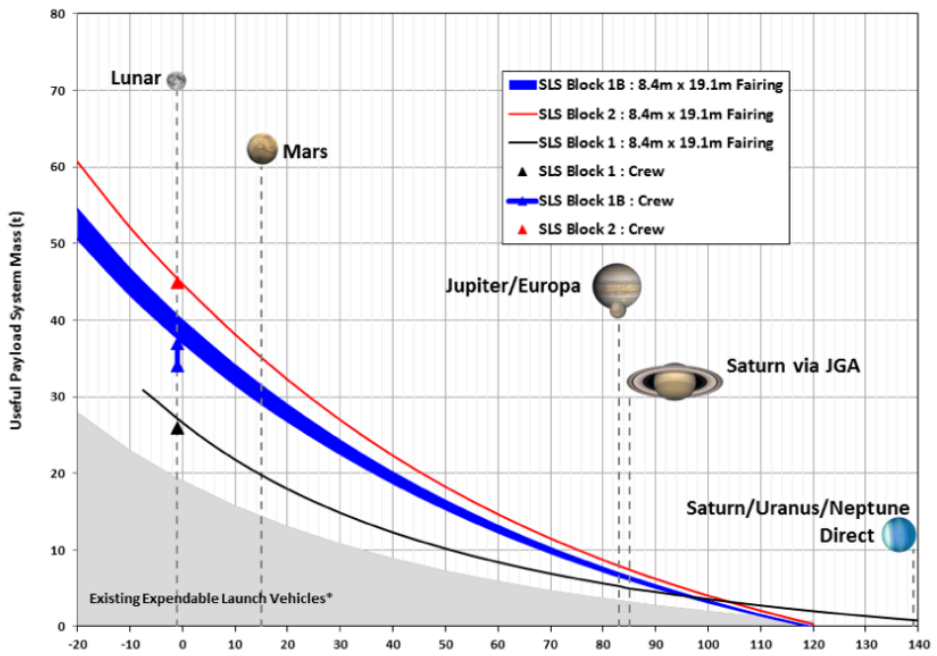

Figure 3. SLS provides superior departure energy, enabling more mass to destination or reduced transit times, depending on mission parameters. 
hardware while taking advantage of the increased volume the large SLS fairings offer. The Block 1B crew vehicle will offer a payload volume of up to $400 \mathrm{~m}^{3}\left(14,126 \mathrm{ft}^{3}\right)$ in its USA for sending co-manifested payloads up to about $9 \mathrm{t}$ to the lunar vicinity. Using SLS to send crew in the Orion vehicle, along with co-manifested and secondary payloads, on a regular cadence of missions to the Gateway will enable NASA to streamline mission design, using one launch to send multiple payloads to deep space.

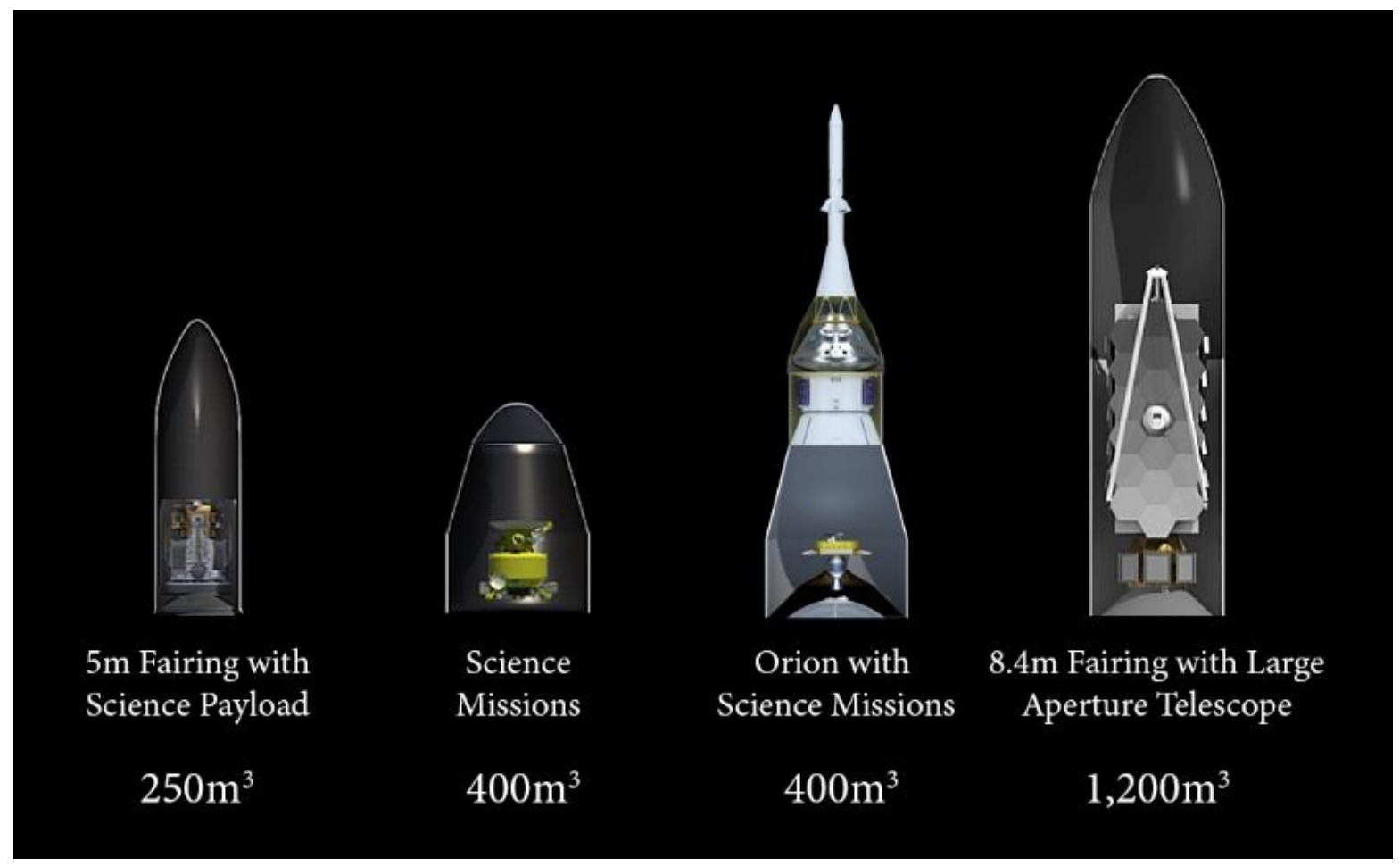

Figure 4. With more fairing volume and greater departure energy, SLS can send a wide array of payloads to lunar orbit or deeper into the solar system.

With $136 \mathrm{U}$ CubeSat payloads manifested on EM-1, NASA is setting a precedent for including secondary payloads on future flights, where mission parameters permit. The OSA in the Block 1 crew vehicle can accommodate 17 berths for $6 \mathrm{U}$ and $12 \mathrm{U}$ smallsats, plus an additional avionics unit to control deployment. For EM-1, mission planners chose to utilize only 13 of the 17 slots because of a mass limitation of 1,000 pounds total for the secondary payloads including the deployment system. Similar to the criteria used to choose the payloads for EM-1, for future flights NASA is interested in payloads that can return data that will help address strategic knowledge gaps in the Agency's plan to sustainably extend human presence to deep space. Block 1B flights may have accommodations for secondary payloads on a payload adapter. User demand for propulsive EELV Secondary Payload Adapters (ESPA)-class payloads is currently being evaluated. Larger payloads, in the 200-300 kg class, and constellations of smallsats are also possible on future flights. Although the primary mission of NASA's deep space exploration system will remain human exploration of deep space and deployment of large-volume payloads, rideshare opportunities for smallsats are expected to be an integral part of future missions.

\section{Progress Toward First Flight}

Shifting NASA's space transportation and operations from the Space Shuttle Program, a reusable vehicle designed for repeat trips to LEO, to a deep space rocket requiring maximum performance to enable the world's most demanding missions, has presented a number of challenges. Yet manufacturing of the Block 1 vehicle for EM-1 is nearing completion, with the ICPS and OSA complete, along with the four RS-25 engines and their upgraded controllers. At the time of writing, the solid rocket motors and the LVSA were close to completion. Forward work on the EM-1 Block 1 build includes completion of the forward and aft booster assemblies as well as the core stage, an all-new development. 


\section{A. Solid Rocket Boosters}

The SLS solid rocket boosters together can generate more than 7.2 million pounds of average thrust, or more than 75 percent of vehicle thrust at liftoff. Similar to the space shuttle boosters, the SLS boosters burn for about two minutes and then are jettisoned. Their design includes three assemblies: forward, solid-fuel motor and aft. To meet SLS mass and trajectory requirements, the motor incorporates substantial design and system upgrades, including an additional propellant segment in each motor, new grain geometry, asbestos-free insulation for safer handling on the ground and a state-of-the art avionics system. The more powerful five-segment motor provides a greater total impulse and takes advantage of Northrop Grumman's (formerly Orbital ATK) experience manufacturing solid rocket motors. The forward assembly on each booster comprises a forward skirt, avionics boxes, frustum, nose cone and four booster separation motors. The forward assembly is also the location for the booster's thrust takeout. Unlike most other launch vehicles that utilize strap-on boosters with thrust takeout structure in the aft area of the launch vehicle, the forward

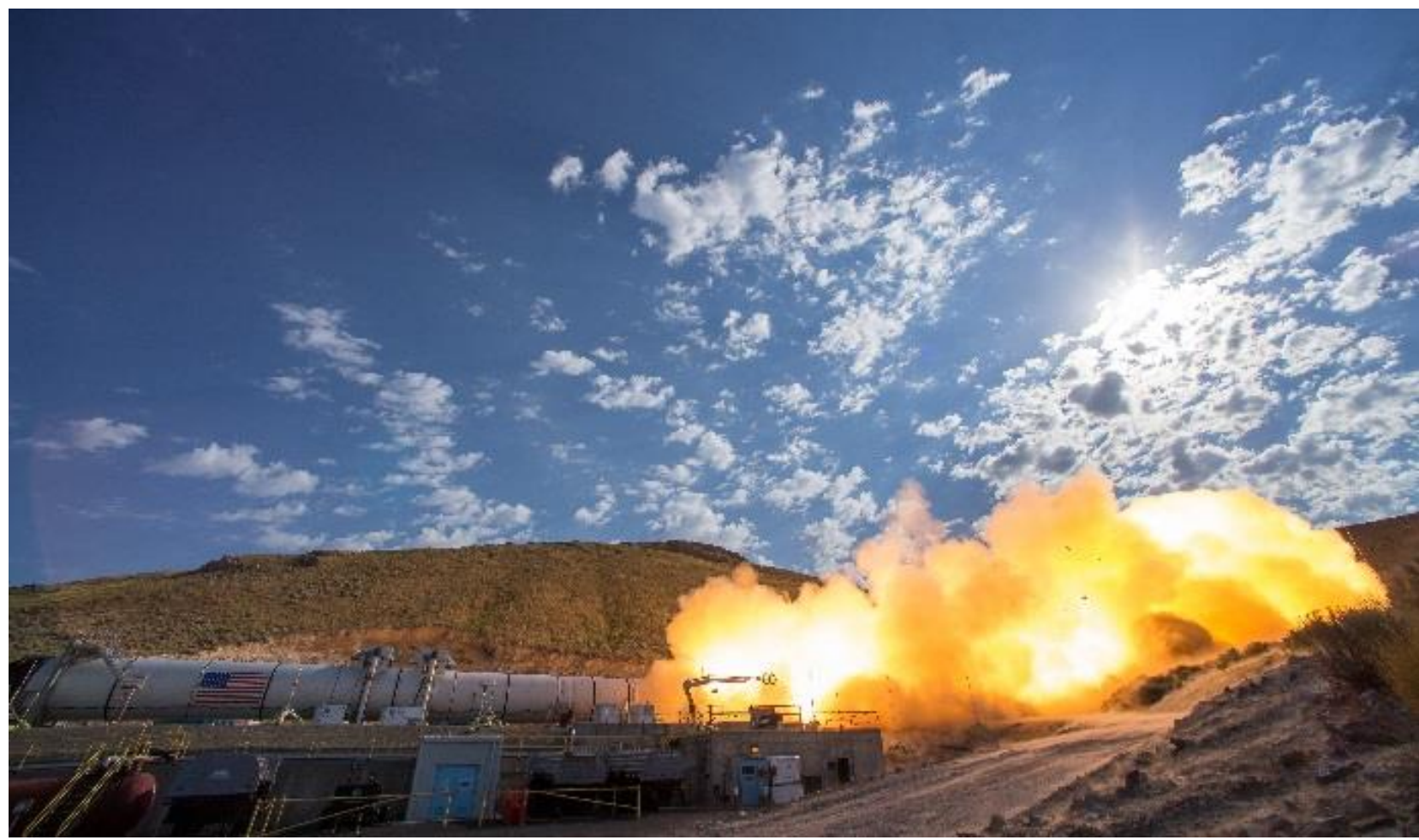

Figure 5. The SLS Program and boosters prime contractor Northrop Grumman have conducted five fullscale static test firings of the five-segment solid motors to support certification for flight.

thrust takeout location is of space shuttle-heritage and helps reduce core stage mass. At the Booster Fabrication Facility (BFF) at KSC, Northrop Grumman has largely completed refurbishment work on the forward assemblies. As of the time of writing, technicians have recently begun applying thermal protection to most of the forward assembly structures. The solid rocket motor is the largest of the three assemblies and includes five motor segments, nozzle, aft exit cone and igniter. For EM-1, the nozzle assemblies are complete, as are seven of the 10 motor segments. Igniter installation is finishing and the remaining three motor segments are in final processing. Five full-scale motors and numerous subscale motors have been fired on the path to flight certification. The final two full-scale hot-fire tests of qualification motors tested motor performance at propellant mean bulk temperatures (PMBTs) of 90 degrees Fahrenheit (32 degrees Celsius) and 40 degrees Fahrenheit (4 degrees Celsius), respectively. Each booster's aft assembly includes an aft skirt, thrust vector control (TVC) system and avionics boxes. Currently, technicians in the BFF are building up the TVC systems in the aft skirts and preparing checkout facilities for final testing of the TVC systems.

\section{B. Engines}

On each SLS flight, the four Aerojet Rocketdyne RS-25 engines will generate more than 512,000 pounds $(232,239$ $\mathrm{kg}$ ) of vacuum thrust each at 109 percent of rated power level (RPL). Main engine cutoff (MECO) will occur about eight minutes into the flight. The Program chose the RS-25 engine (formerly known as the Space Shuttle Main Engine 
[SSME]) based on power, successful performance over 135 space shuttle missions and the engine's well-understood characteristics over more than a million seconds of ground and flight operating time. The SLS Program has 16 engines remaining from the space shuttle era, which will be used on the first four SLS launches. SLS will operate them at higher power levels and pressures and in a more severe thermal environment than during space shuttle flights to LEO. The four EM-1 engines have been certified to fly in these more extreme environments and have been upgraded with state-of-the-art engine controllers, all of which have been hot-fired on adaptation engines at NASA's Stennis Space Center (SSC).

Beginning with the fifth flight, SLS will incorporate newly built RS-25 engines. New builds of the engine will be certified to operate at 111 percent of the original SSME RPL of 375,000 pounds of thrust at sea level, 470,000 pounds thrust at vacuum. In addition, the new production series incorporates numerous improvements to streamline manufacturing, reduce costs and compress schedule. Several of the parts on the new engine series use technological innovations such as additive manufacturing. A 3D-printed pogo accumulator was hot-fire tested at SSC earlier in 2018 and more tests are on tap to characterize the additively manufactured parts before they are used in new-build RS-25 engines on future SLS flights.

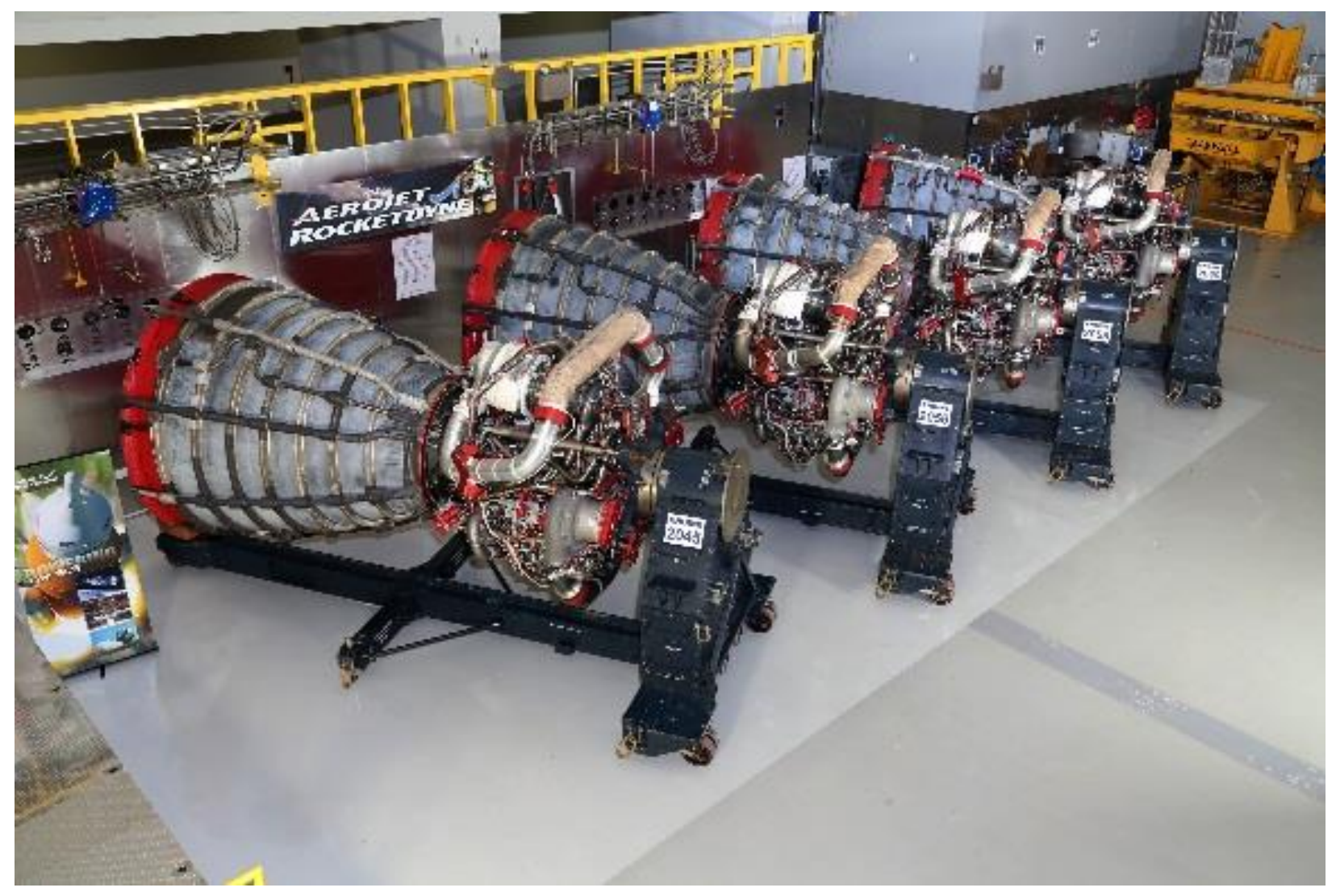

Figure 6. The four RS-25 engines for the first mission, Exploration Mission-1 (EM-1), have been upgraded with state-of-the-art computerized controllers and are ready for integration with the core stage. 


\section{Core Stage}

The all-new 212 foot $(64.6 \mathrm{~m})$ core stage — the largest ever built for flight in terms of volume and height — is the backbone for the rocket, with flight computers contained in the forward skirt. The stage is comprised of five primary structures from forward to aft: a forward skirt, a LOX tank, an intertank structure, the LH2 tank and an engine section.

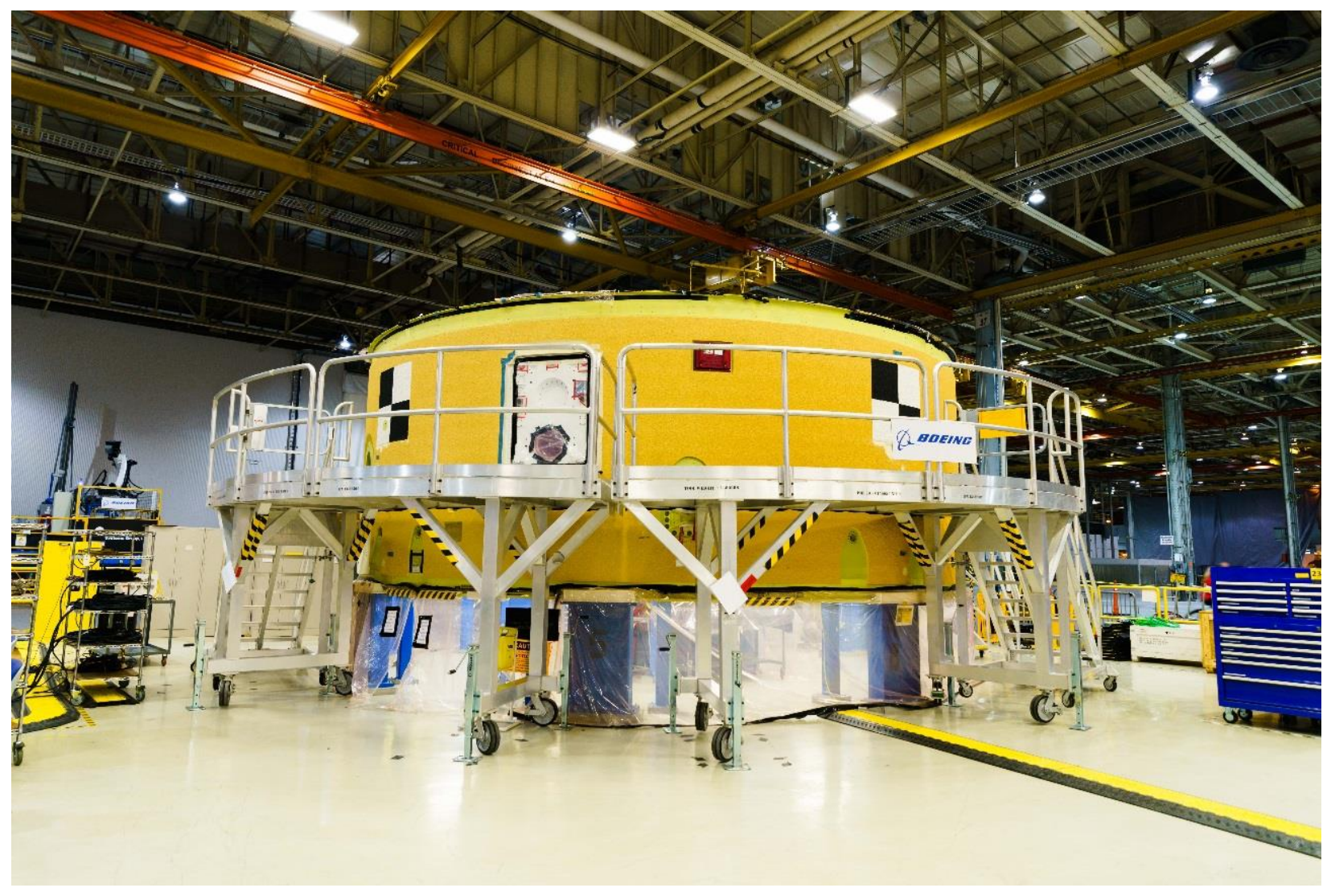

Figure 7. Core stage prime contractor Boeing recently completed work on the forward skirt component of the 212-foot $(64.6 \mathrm{~m})$ core stage at Michoud Assembly Facility.

The intertank, which holds the forward attach points for the solid rocket boosters, has been constructed by bolting together (rather than welding) barrel panels for the strength required to accommodate booster thrust. The other four structures were assembled using the Vertical Assembly Center (VAC), the world's largest spacecraft welding tool. At the time of writing, the avionics in the forward skirt had been powered on and that component was complete and ready to be mated with the LOX tank and intertank. Boeing continues to make progress finishing the other core stage components for flight. Major subassembly and integration work is underway in the engine section. Technicians are installing the TVC system, boat tail assemblies, pumps, manifolds, ducts, cables, harnesses and instrumentation for flight. The LH2 tank, which will hold 537,000 gallons (2,032,766 l) of cryogenic fuel, has been proof-tested, cleaned and primed. After priming, technicians will apply the thermal protection system (TPS), similar to the TPS used on the space shuttle's external tank. The EM-1 LOX tank is currently being sprayed with TPS. Robotic acreage sprays are possible on the barrel section, but manual spraying is required for the domes.

\section{Upper stage and adapters}

The ICPS will supply in-space propulsion for the Block 1 vehicle. Manufactured by Boeing and ULA in Decatur, Alabama, USA, the stage is an LH2/LOX system and uses one Aerojet Rocketdyne RL10B2 engine. The ICPS for EM-1 was completed in 2017 and delivered to the EGS Program at KSC. Modifications to the ULA DCSS to meet SLS mission requirements include lengthening the LH2 tank, adding hydrazine bottles for attitude control and some minor avionics changes. 
In addition to the ICPS, the upper part of the rocket includes two adapters: the LVSA and the OSA. The LVSA connects the core stage to the ICPS and also partially encloses the ICPS. The LVSA is nearly finished, with technicians and engineers at the time of writing installing a pneumatic actuation system and mating the adapter to a frangible joint assembly. The other adapter, the OSA, will house the $136 \mathrm{U}$ secondary payloads and provide a connection between

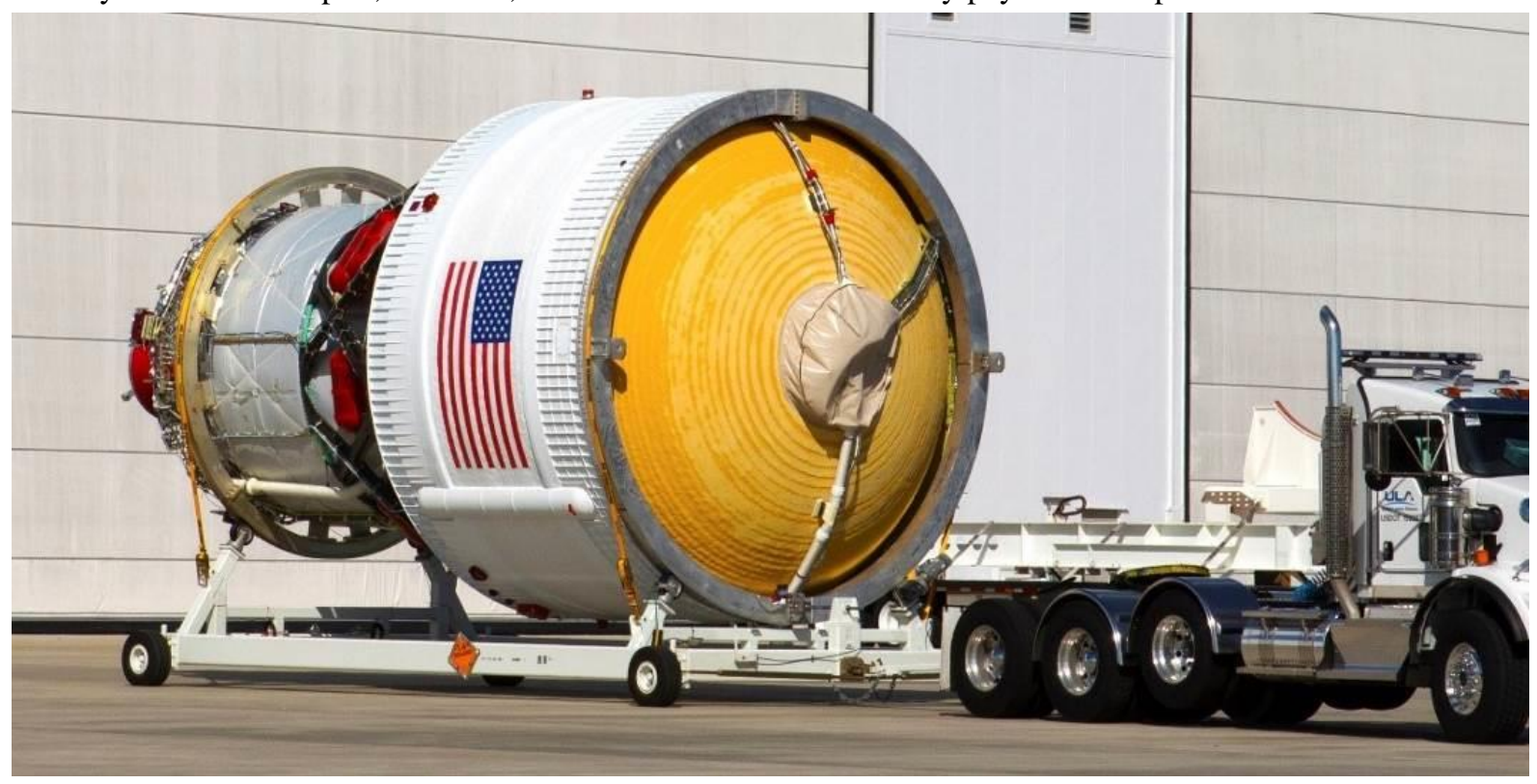

Figure 8. United Launch Alliance (ULA) and Boeing delivered the completed Interim Cryogenic Propulsion Stage (ICPS) to the Exploration Ground Systems (EGS) Program in 2017.

the ICPS and a spacecraft adapter located aft of Orion's service module. The SLS Program provided a secondary payload deployment system, consisting of an avionics unit and mounting brackets, inside the OSA. Payload developers purchased a specified commercial off-the-shelf (COTS) dispenser. Payloads will be integrated into the COTS deployers prior to stacking the vehicle at KSC. The OSA is complete and was shipped to EGS in early 2018. The SLS Program's Spacecraft Payload Integration and Evolution Office (SPIE) manages the upper stage of the rocket, including payload accommodations and adapters.

\section{E. Structural testing}

Because SLS is being designed from the ground up for human exploration, a key safety requirement is testing full-scale hardware for structural qualification. Real-world data gained from structural testing enables engineers to verify analytical models. As a result, the largest structural test campaign since the Space Shuttle Program is underway at MSFC to ensure SLS elements meet the stringent environments and loads the vehicle will experience during launch and ascent.

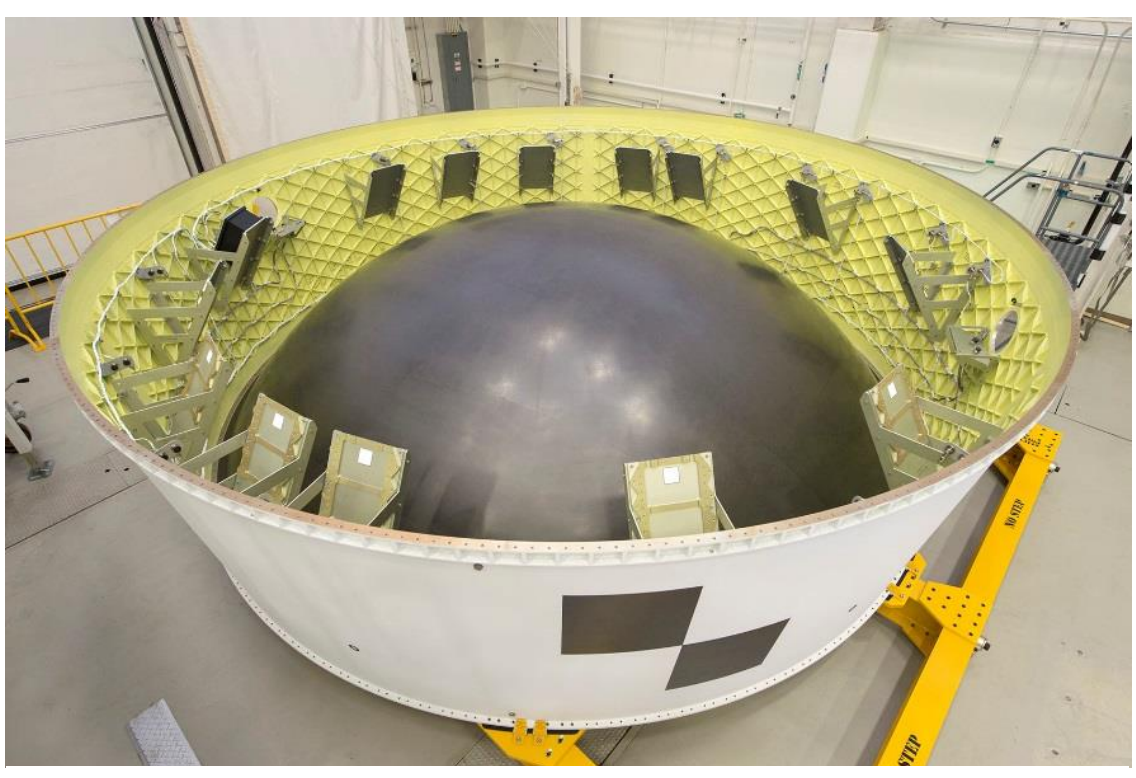

Figure 9. The completed EM-1 Orion Stage Adapter (OSA), showing the internal diaphragm and accommodations for $136 \mathrm{U}$ CubeSat secondary payloads before the adapter shipped to the EGS Program at KSC. 


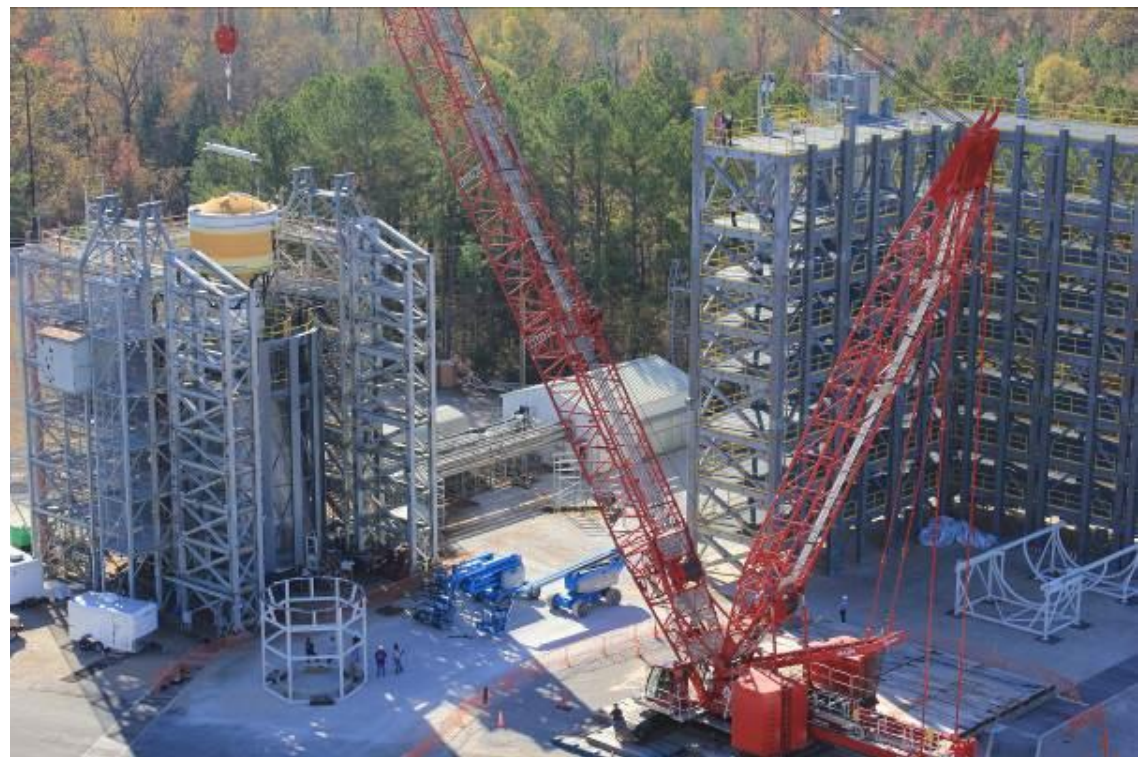

Figure 10. The Integrated Structural Test (IST) applied loads up to 40 percent greater than flight to full-scale upper stage structural test articles to support qualification for flight.

Engineers at MSFC first tested the upper stage and payload sections of SLS in a test series called the Integrated Structural Test (IST). For this test, flight-like components for the upper part of the rocket were stacked in a test stand from bottom to top: a core stage simulator, the LVSA, a frangible joint assembly, the ICPS, the OSA and an Orion simulator. Together, they formed a test stack $62 \mathrm{ft} .(19 \mathrm{~m})$ tall and $28 \mathrm{ft} .(8.5 \mathrm{~m})$ wide at the widest point. For this test series, loads up to 40 percent greater than flight loads were applied to the stack. Twenty-eight pistons applied compression, tension, bending, torsion and shear loads. Engineers reviewed data collected through 1,900 channels. The IST was

successfully completed in May 2017.

The first core stage structural test article (STA), the engine section STA, shipped from Michoud, where it was manufactured, to MSFC in 2017; testing on that article is also complete. The engine section STA was installed in a unique 50 feet $(15 \mathrm{~m})$ test stand and connected to more than 50 hydraulic actuators. During the test series, the actuators simulated more than 3 million pounds of upward RS-25 engine thrust loads and up to 750,000 pounds of loads on each side for the outward forces created by the boosters. A series of tests also validated brackets designed to hold feedlines from the LOX tank. Engineers recorded and analyzed more than 3,000 channels of data for each test case to verify the capabilities of the engine section and LOX feedlines.

The next core stage STA to arrive at MSFC, the intertank STA, consists of a structurally flight-like intertank with two simulators that mimic the LH2 and LOX tank structures. At MSFC, the intertank STA has been installed in the test fixture and qualification testing began in July 2018. The first tests scheduled include 21 influence (50 percent loads) test cases, including one cryo test case, which has been completed, and 20 mechanical test cases. Limit (100 percent loads) and ultimate (140 percent loads) test cases are scheduled to be completed by the end of 2018. Approximately 100 load actuators, ranging in size from 10 to $18 \mathrm{in}$. (25 to $46 \mathrm{~cm}$ ) bore cylinders are applying the loads to the intertank STA. The LH2 and LOX STAs will be tested at MSFC at a later date.

\section{Manufacturing Lessons Learned}

With work complete on several elements of the first Block 1 build, NASA and its contractors are already at work procuring elements and manufacturing the second Block 1 vehicle, currently slated to fly either the first crewed mission, EM-2, or the first cargo flight, SM-1. Many efficiencies have already been realized when fabricating the second Block 1 vehicle; this section will focus on lessons learned manufacturing the LVSA and OSA, which were welded in MSFC's Advanced Weld Facility using a modular tooling adaptation to the robotic weld tool.

Technicians at MSFC's Advanced Weld Facility have constructed the EM-1 LVSA and OSA as well as full-scale test hardware for the IST (see Section IV, Part E). The LVSA comprises two cones and two ring sections. The process for fabricating the final cone-shaped LVSA consists of first vertically welding panels using a friction-stir weld executed on MSFC's vertical weld tool to fabricate the bottom cone, then repeating that process to fabricate the upper cone. Next, a circumferential friction-stir weld is performed using the robotic weld tool, which joins the two smaller cones to create the final cone, which measures 27.5 feet $(8.4 \mathrm{~m})$ tall and tapers from 27.5 feet $(8.4 \mathrm{~m})$ at the bottom (diameter of the core stage) to 16.5 feet $(5 \mathrm{~m})$ at the top (diameter of the ICPS). The final manufacturing steps consist of using the robotic weld tool to weld the two rings onto the top and bottom of the joined cone to create the final coneshaped structure. 
The angled sides of the two cones must align perfectly in order to create a strong weld (friction-stir welds are typically 10 percent stronger than traditional welding). To support the cones on the robotic weld tool, a single-use superstructure tooling fixture that would sit on the welding tool's turntable was first used to manufacture the OSA for Exploration Flight Test-1 (EFT-1), a test flight of the Orion spacecraft that launched in 2014. This traditional tooling proved to be bulky, expensive and time-consuming to set up and disassemble. In addition, it was configurationspecific, usable only to create a single piece of hardware. Engineers searching for a way to streamline production for future manufacturing developed the idea of modular tooling that could be installed in the turntable and adaptable to a number of geometries, including the cones that comprise the greater portion of the LVSA. This modular tooling, consisting of columns of varying heights that fit in the t-grooves on the turntable and supporting rings attached to the back of the pillars, along with several other key pieces of the system, enabled technicians to quickly set up tooling to support the LVSA and the OSA. The system is flexible, modular, re-usable and efficiently dismantled and requires less storage space when not in use.

Another innovation enabled the cones to be welded together using the modular tooling on the robotic weld tool. Hawthorne clamps, first used to manufacture the Saturn rockets during the Apollo program, held the two cones securely together at a perfectly aligned angular interface around the circumference of the join on the outside, while

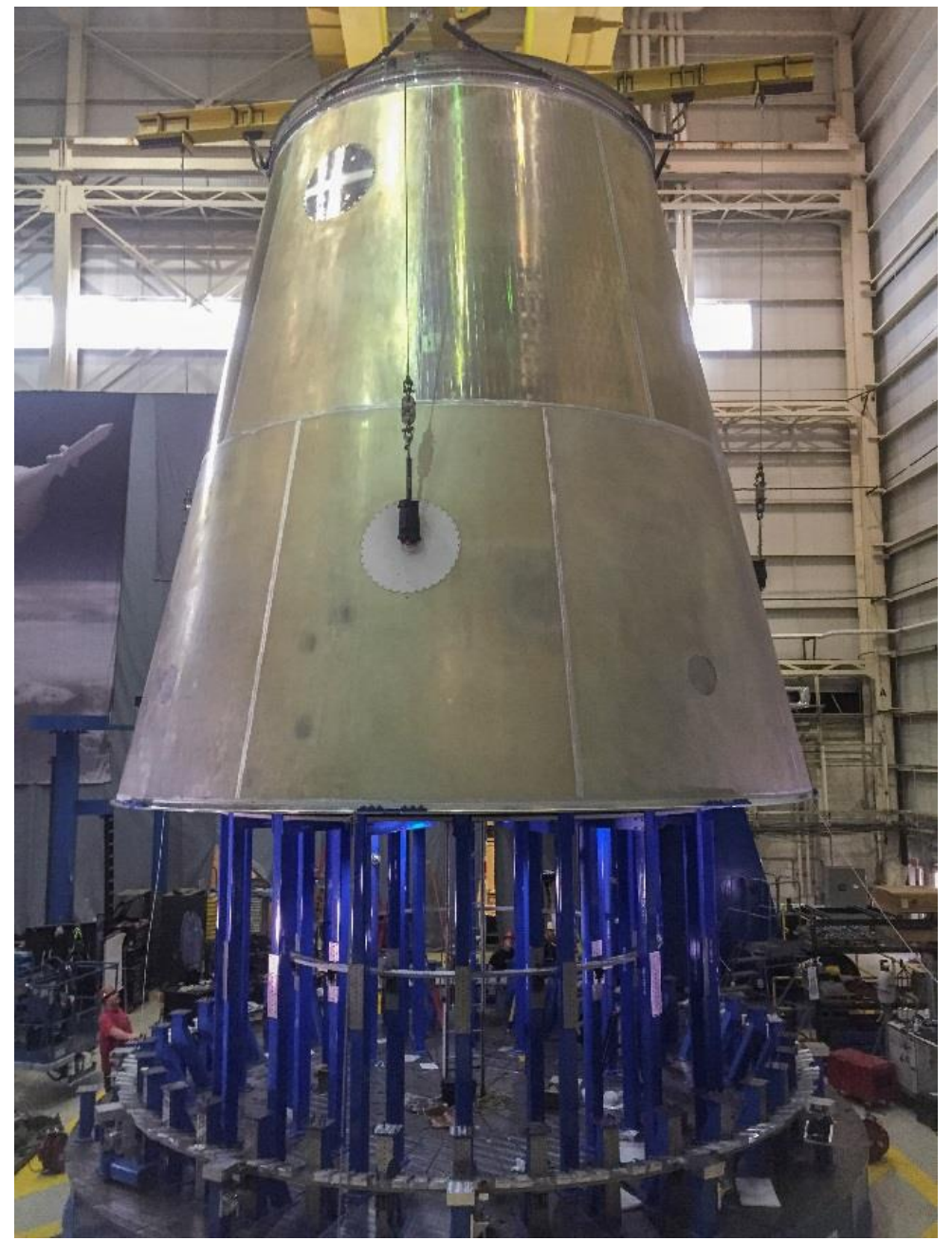

Figure 11. Design and installation of modular tooling (blue), consisting of columns of varying heights that can moved to varying locations on the robotic weld tool turntable at Marshall Space Flight Center (MSFC), gives technicians a reusable tool to weld complex geometries. the moveable modular structure supported the inside of the two cones. The Hawthorne clamps consist of two parts. As technicians aligned the two cones they machined a slot and fed a stainless steel ribbon from one half of the Hawthorne clamp to a pickup on the other half. Then, they tightened the two halves of the clamp to securely hold the interface between the two cones together. Technicians then performed a fusion tack weld to complete the welding process. A cutter broke the ribbon in the clamp and the two halves of the Hawthorne clamps fell away. Re-use of the Hawthorne clamps, a proven successful tool from the manufacture of NASA's Saturn V, enabled use of the new, innovative modular tooling. This synergistic approach between new tooling and heritage knowledge substantially improved the schedule in welding the EM-1 LVSA, reducing assembly from six months to one month. In addition, the Program saved more than $\$ 5$ million over purchasing single-use tooling to weld the LVSA. Welding the OSA is a simpler process but use of the modular tooling to weld the flight hardware also resulted in significant cost savings and schedule reduction. Two of the SLS Program's goals are to create a flexible launch vehicle solution to support a range of deep space missions while keeping affordability in mind. Using these two approaches, technicians were able to achieve 430 yards $(393 \mathrm{~m})$ of defect-free friction-stir welds and the modular tooling and critical process knowledge is in place to manufacture the next adapters for the next Block 1 flight. 


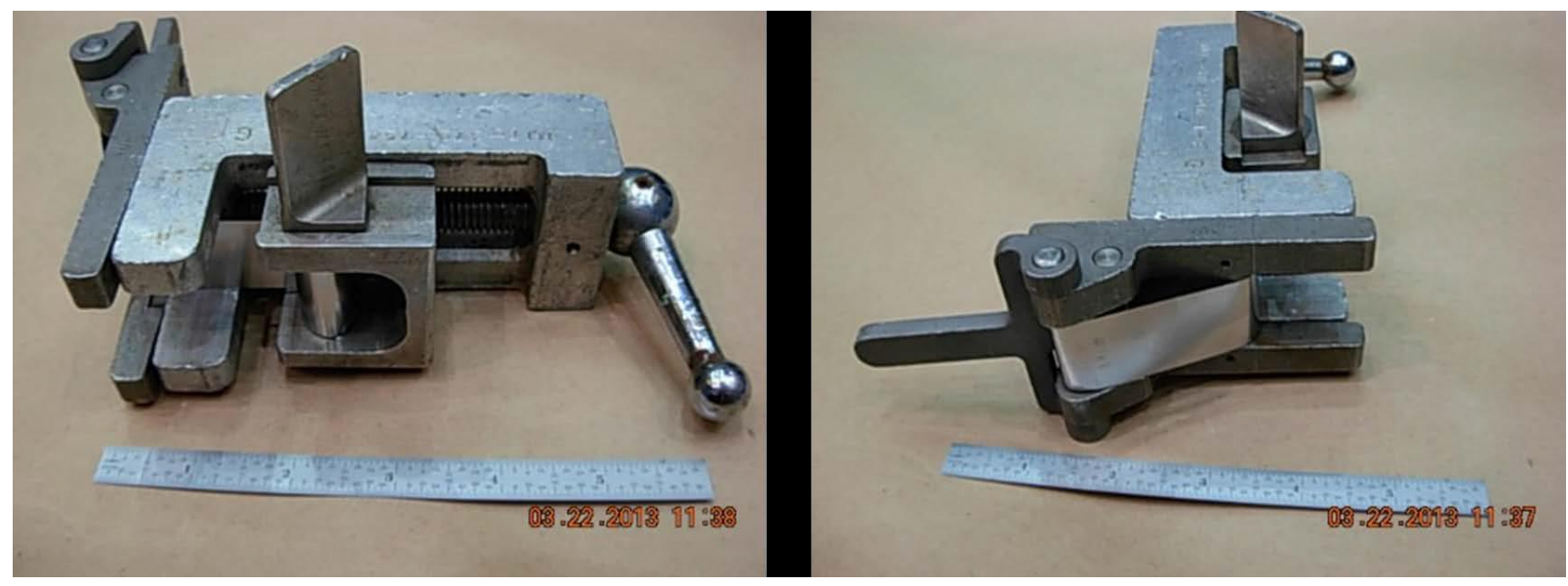

Figure 12. Hawthorne clamps, used to manufacture the Saturn launch vehicles decades ago, provided a solution for securely fastening and vertically aligning the two cones of the Launch Vehicle Stage Adapter (LVSA) so the robotic friction-stir welding tool at MSFC could execute defect-free welds.

\section{Conclusion}

Shifting NASA's human spaceflight program from a LEO paradigm to deep space requires a super heavy-lift launch vehicle, a crew vehicle that's more capable that any ever built before and upgraded ground processing and launch facilities at KSC - America's multiuser spaceport. The SLS vehicle provides unparalleled power and payload capacity to launch the world's most critical, strategic missions to deep space. A family of rockets is planned with crew and cargo configurations available in Block 1, Block 1B and Block 2 variants. The Block 1 cargo vehicle will feature a $5 \mathrm{~m}$-class fairing; larger $8.4 \mathrm{~m}$ fairings in varying lengths will give the Block 1B and Block 2 cargo vehicles unparalleled volume for strategic payloads. In addition, mission planners hope to offer CubeSat developers rideshare opportunities on flights with excess capacity. Secondary payload accommodations in $6 \mathrm{U}, 12 \mathrm{U}$ or other sizes may be on the OSA (Block 1) or on a payload adapter (Block 1B).

The first vehicle, in the Block 1 crew configuration, is nearing completion, with RS-25 engines, the ICPS and the OSA all complete. In addition, the fivesegment solid rocket motors are nearly complete and refurbishment of the booster forward and aft assemblies is expected to finish in early 2019 . The first core stage component, the forward skirt, is complete and will be joined with the other forward components soon. The core stage, an all-new development, is rapidly being outfitted for flight and then will be assembled with the RS-25 engines. The second Block 1 build is already well underway, and increased efficiencies are already evident, stemming from lessons learned in manufacturing the EM-1 vehicle.

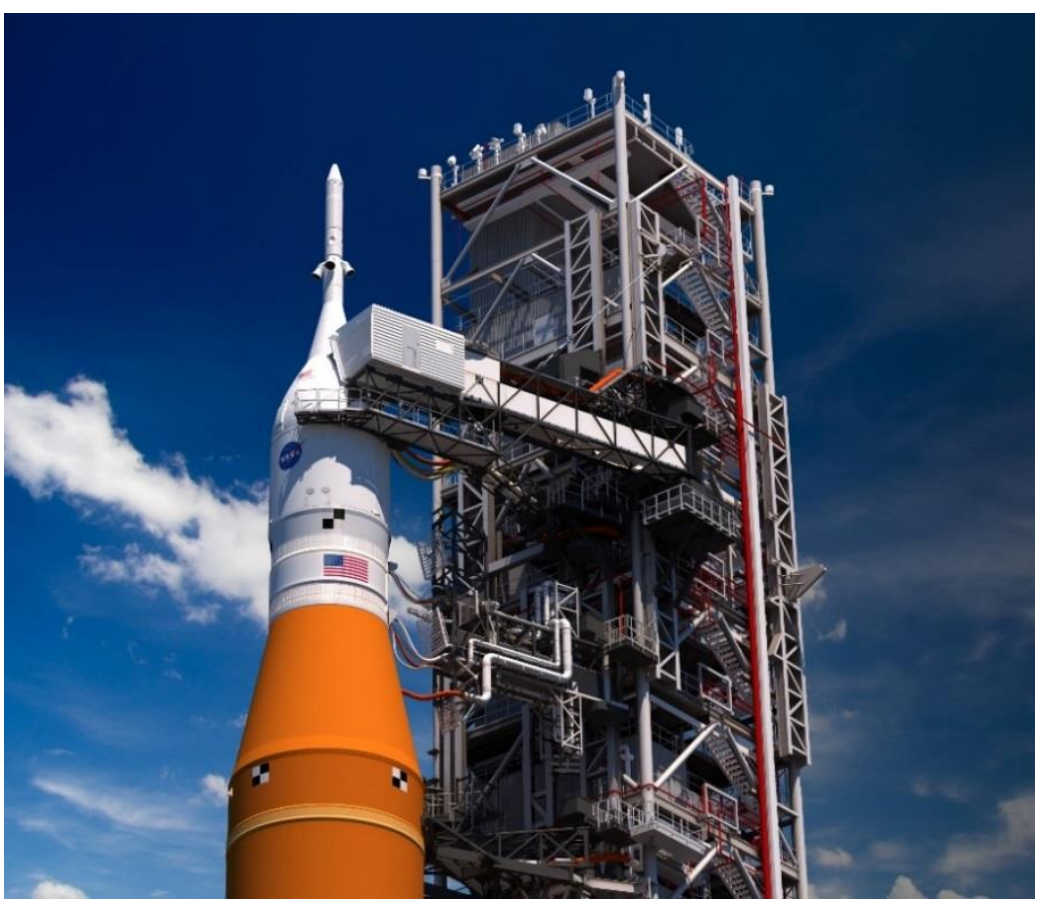

Figure 13. NASA's deep space exploration system, including SLS, Orion and ground processing and launch facilities at $\mathrm{KSC}$, will usher in a new era of human exploration of the Moon and beyond. 